\title{
Estudo da estrutura fatorial e das qualidades psicométricas da versão portuguesa da Male Body Attitude Scale-Revised
}

\author{
Male Body Attitudes Scale-Revised: Factor structure and psychometric properties of the Portuguese version
}

Artigo Original | Original Article

\section{Cláudia Ferreira PhD (1a), Joana Marta-Simões Estudante PhD (1b), Sara Oliveira ScM (1c), João Duarte Psy (1d)}

(1) CINEICC, Centro de Investigação em Neuropsicologia e Intervenção Cognitivo-Comportamental da Faculdade de Psicologia e de Ciências da Educação da Universidade de Coimbra, Portugal

(a) Revisão da literatura; Tratamento e discussão dos dados; Redação do manuscrito.

(b) Contributo para a obtenção e adaptação do instrumento de medida; Revisão da redação final do manuscrito.

(c) Tratamento dos dados; Contributo na redação do manuscrito.

(d) Recolha e inserção dos dados para análise estatística; Contributo para a revisão da literatura.

Autor para correspondência | Corresponding author: Cláudia Ferreira; Rua do Colégio Novo, Apartado 6153, 3001-802, Coimbra, Portugal; claudiaferreira@fpce.uc.pt

\section{Palavras-Chave}

Análise confirmatória Imagem corporal Instrumentos de medida

Massa muscular

Massa gorda

Sexo masculino

\section{RESUMO}

Objetivo: A literatura demonstra que a imagem corporal é uma dimensão central de auto e heteroavaliação e um foco de preocupação não só para as mulheres, mas também para os homens. Diversos autores têm sublinhado a necessidade de um maior investimento no desenvolvimento e validação de instrumentos de medida para a imagem corporal para o sexo masculino, dado que homens e mulheres se distinguem significativamente em relação às preocupações associadas à aparência física. A Male Body Attitude ScaleRevised (MBAS-R) é a versão revista de um instrumento de medida (MBAS), especificamente desenvolvido para a população masculina, que visa avaliar as atitudes e preocupações em relação à imagem corporal. O presente estudo visa estudar a estrutura fatorial e as propriedades psicométricas da versão portuguesa da MBAS-R.

Métodos: $\mathrm{O}$ estudo foi conduzido numa amostra constituída por 222 homens da população geral portuguesa, com idades compreendidas entre os 18 e os 65 anos.

Resultados: Os resultados de uma série de análises fatoriais confirmatórias demonstraram a adequação da versão portuguesa da MBAS-R (12-itens) com uma estrutura de dois fatores (massa muscular e massa gorda). A análise dos itens da versão portuguesa da MBAS-R revelou que todos os itens contribuem consistentemente para a respetiva subescala e para a medida global. Adicionalmente, tanto a escala total como as subescalas da versão portuguesa da MBAS-R revelaram boa consistência interna. Os resultados mostraram ainda que as preocupações e atitudes em relação à imagem corporal, avaliadas pela MBAS-R, se associam positivamente a vergonha externa, vergonha corporal e inflexibilidade alimentar.

Conclusões: Os resultados sugerem que a MBAS-R é uma medida breve e válida para avaliar e caraterizar as preocupações masculinas em relação à imagem corporal de um modo global e, simultaneamente, especificamente em relação a duas dimensões centrais da vivência da imagem corporal (massa muscular e gordura corporal) nesta população.

\section{Keywords}

Confirmatory analysis

Body image

Assessment measures

Muscularity

Body fat

Men

\section{ABSTRACT}

Objectives: Literature has shown that body image is a central dimension of self- and hetero-evaluation, and a focus of concern, not only for women but also for men. Several authors have argued the need for more significant investment in the development and validation of assessment measures of male body image, given that men and women are significantly different regarding their body image concerns. The Male Body Attitude Scale-Revised (MBAS-R) was explicitly designed for the male population and aims to evaluate attitudes and concerns regarding body image. The present study aimed at studying the factor structure and psychometric properties of the Portuguese version of MBAS-R.

Methods: The present study used a sample composed of 222 men form the general male Portuguese population, aged between 18 and 65 .

Results: Results of a series of confirmatory factor analysis showed the adequacy of the Portuguese version of MBAS-R (12-items), with a two-factor structure (muscularity and body fat). The analysis of the items of the Portuguese version of MBAS-R revealed that all items consistency contributes to the respective subscale, as well for the global measure. Additionally, both the global scale and its subscales revealed good internal consistency. Results also showed that body image attitudes and concerns measured by the MBAS-R associated positively with external shame, body image shame, and eating inflexibility.

Conclusions: Results suggest that the MBAS-R is a brief and valid measure to assess and characterize male body image concerns, both globally and specifically, i.e., regarding two different central dimensions of body image in this population (muscularity and body fat). 


\section{INTRODUÇÃO}

A investigação no âmbito da imagem corporal tem-se focado quase exclusivamente em estudos com amostras de mulheres, no entanto as evidências apontam para um crescente interesse pela compreensão da vivência da imagem corporal nos homens (Cain, Epler, Steinley, \& Sher, 2011; Yean et al., 2013). De facto, historicamente as preocupações e/ou insatisfação com a aparência física têm sido reconhecidas como problemas ou dificuldades associadas à população feminina e ao seu desejo de perder peso ou ficar mais magras (Daniel \& Bridges, 2010; Pope, Phillips, \& Olivardia, 2000). No entanto, os dados empíricos parecem suportar que a imagem corporal é um foco de preocupação e uma dimensão central na auto e heteroavaliação tanto para as mulheres como para os homens (McCabe \& Ricciardelli, 2001).

Os estudos documentam que na população do sexo masculino as dificuldades associadas à imagem corporal estão associadas a comportamentos maladaptativos em relação à aparência física e comportamento alimentar, com impacto negativo significativo ao nível psicológico, social e da saúde física (Calogero, 2009; Dakanalis \& Riva, 2013; Dakanalis et al., 2015; Sepúlveda et al., 2016). Entre os efeitos negativos consequentes da adoção de estratégias de controlo do peso e da massa muscular no sexo masculino, tem sido reportado um acréscimo do comportamento alimentar inflexível e perturbado e do uso de esteroides anabolizantes androgénicos e de suplementos alimentares (Smith, Hawkeswood, Bodell, \& Joiner, 2011), assim como consequentes problemas de saúde física (tais como problemas renais e hepáticos) (Olivardia, Pope, Borowiecki, \& Cohane, 2004) e um decréscimo da saúde mental (e.g., altos níveis de depressão e ansiedade social, baixa autoestima) (e.g., Cafri, Strauss, \& Thompson 2002; McCreary \& Sasse, 2000).

Pela sua importância, estes dados motivaram um interesse crescente no estudo das preocupações e dificuldades associados à imagem corporal nos homens, e da forma como estas dificuldades poderão ter um impacto negativo na saúde física e ajustamento psicológico desta população (Dakanalis \& Riva, 2013; Olivardia et al., 2004; Stanford \& McCabe, 2002). No entanto, na avaliação e estudo das atitudes e preocupações com a imagem corporal nos homens são consistentemente usadas medidas desenvolvidas com base em modelos teóricos desenhados para mulheres, prática considerada inapropriada (Cafri \& Thompson, 2004; Ryan, Morrison, Roddy, \& McCutheon, 2011). A consideração da aplicação destes instrumentos a sujeitos do sexo masculino como inapropriada deve-se fundamentalmente ao facto de homens e mulheres se distinguirem qualitativamente em relação às suas preocupações com a imagem corporal (Tylka, Bergeron, \& Schwartz, 2005). Mais especificamente, os estudos mostram que a maioria dos homens expressa o desejo de ganhar massa muscular e perder massa gorda, enquanto as mulheres habitualmente reportam o desejo de perder peso, perder volume e de terem uma silhueta mais filiforme (Cafri \& Thompson, 2004; Ridgeway \& Tylka, 2005). Assim, mais do que a avaliação do grau de (in)satisfação em relação ao peso corporal, na população do sexo masculino são o grau de (in)satisfação em relação à massa muscular e massa gorda que devem ser considerados pelos instrumentos que avaliam a imagem corporal (e.g., Blashill, 2010; Cohane \& Pope Jr., 2001). Adicionalmente, os homens tendem a reportar insatisfação em relação a áreas corporais diferentes das reportadas como primordial foco de preocupação ou de avaliação negativa da maioria das mulheres (Dakanalis \& Riva, 2013; McCabe \& Ricciardelli, 2001). Particularmente, os homens tendem a reportar o desejo de ganhar massa muscular na parte superior do corpo (e.g., braços, peito e costas), enquanto nas mulheres o desejo de controlar ou alterar a sua aparência tende a focar-se na perda de peso e/ou volume na zona inferior do corpo (tal como ancas, coxas e nádegas) (e.g., Dakanalis \& Riva, 2013).

A maioria dos instrumentos de avaliação da imagem corporal, tais como Body Satisfaction Questionnaire (BSQ; Cooper, Taylor, Cooper, \& Fairbum, 1987), as subescalas de procura da magreza e insatisfação corporal do Eating Disorder Inventory (EDI; Garner, Olmsted, \& Polivy, 1983) ou as subescalas de preocupação com o peso e preocupação com a forma corporal da Eating Disorders Examination-Questionnaire (EDE-Q; Fairburn \& Beglin, 1994), são constituídos por itens desenvolvidos para medir a insatisfação com o peso e formas corporais e, mais especificamente, com o desejo de perder peso e de ter uma silhueta mais delgada (Cohane \& Pope Jr., 2001). No entanto, devido às diferenças de género em relação à perceção da forma corporal ideal, aquelas que são consideradas medidas de ouro para a avaliação da imagem corporal nas mulheres não são medidas válidas para populações do sexo masculino (Cafri \& Thompson, 2004). Surge, assim, como imperativo um maior investimento no desenvolvimento e validação de instrumentos de medida para a imagem corporal desenvolvidas especificamente para o sexo masculino.

Em 2005, Tylka et al. (2005) desenvolveram a Male Body Attitudes Scales (MBAS) para medir as preocupações e as atitudes masculinas em relação à aparência física, atendendo às dimensões mais salientes 
e associadas a diferentes indicadores de bem-estar psicológicos nos homens. Assim, a MBAS foi composta por três subescalas: (i) massa muscular, que avalia o grau de (in)satisfação do indivíduo com a sua massa muscular, relativamente a diferentes zonas do seu corpo, (ii) massa gorda, que avalia a perceção e as atitudes pessoais em relação à massa gorda (ou gordura corporal), tanto em termos globais como em relação a áreas específicas do corpo, e (iii) altura que mede o grau de (in)satisfação com a sua altura e o desejo de ser mais alto. Os autores originais (Tylka et al., 2005) consideram que esta composição da escala em diferentes subescalas possibilita uma leitura compreensiva da insatisfação corporal atendendo às especificidades da população masculina, e permite, ainda, que investigadores e clínicos optem pelo uso do total da escala para estimarem um valor global das atitudes em relação à imagem corporal, ou pelo uso de uma das subescalas para avaliarem domínios ou atitudes específicas. Os resultados dos estudos originais confirmaram a adequação da estrutura fatorial da MBAS e boas qualidades psicométricas, revelando adequada consistência interna (com valores de Cronbach que variam entre 0,90 e 0,91 para a escala total; entre 0,89 e 0,91 para a subescala massa muscular, entre 0,90 e 0,94 para a subescala de massa gorda e entre 0,66 e 0,88 para a subescala altura), assim como evidências de boa validade convergente e divergente e estabilidade temporal (Tylka et al., 2005).

Posteriormente, numa amostra de homens homossexuais, Blashill e Vander Wal (2009) reportaram a ambiguidade dos resultados relativos à subescala altura da MBAS e testaram um modelo com dois fatores/subescalas (insatisfação em relação à muscularidade e massa gorda), a qual demonstrou forte validade fatorial. A adequação desta estrutura bidimensional da MBAS foi ainda confirmada para a população adolescente (Sepúlveda et al., 2016). A proposta da MBAS composta unicamente pelas subescalas que avaliam a insatisfação em relação à massa muscular e à massa gorda parece também ser suportada pelos autores originais da medida, os quais consideraram a subescala altura questionável (Tylka et al., 2005).

Embora diversos estudos suportem a validade e a adequação da MBAS, Ryan et al. (2011) consideram que aspetos conceptuais e de estratégia analítica que presidiram o desenvolvimento e a validação desta medida são discutíveis. Por um lado, Ryan et al. (2011) consideram que a versão original da MBAS possui itens problemáticos ou ambíguos, enquanto outros apresentam cross loading em fatores/dimensões diferentes. Paralelamente, estes autores salientam que o facto da subescala que avalia as atitudes face à altura ser constituída por apenas por dois itens traduz uma fragilidade, uma vez que é recomendado um mínimo de 3 a 5 itens por fator/dimensão (Ryan et al., 2011). Finalmente, Ryan et al. (2011) recomendam que o formato de resposta dos itens deveria ser alterado da escala de resposta de 6 para 5 pontos, uma vez que consideram não existir distinção clara entre as opções Usually e Often. Estes aspetos problemáticos motivaram Ryan et al. (2011) a construir uma versão revista desta medida (MBAS-R) constituída por 15 itens, na qual foram: eliminados itens que demonstravam fraca qualidade psicométrica, revisto o conteúdo e a redação de alguns dos itens, adicionados itens para a subescala "altura" e excluída uma das opções de resposta. Os resultados do estudo de Ryan et al. (2011) sugeriram que a MBAS-R (composta por de três fatores) é uma medida válida e robusta, e de um bom indicador das atitudes e preocupações dos homens em relação à sua aparência física. No entanto, os seus autores salientam a necessidade de estudos adicionais que explorem a adequação da estrutura da escala através de análise fatorial confirmatória e corroborem a validade da medida em amostras distintas (Ryan et al., 2011).

O presente estudo pretende validar a MBAS-R para português europeu, explorar a estrutura fatorial mais adequada e examinar as suas propriedades psicométricas. Especificamente, os objetivos do presente estudo foram: (a) explorar a estrutura fatorial de três fatores e de dois fatores (e.g., com a eliminação da subescala altura) numa amostra da população portuguesa do sexo masculino, através de análises fatoriais confirmatórias; (b) examinar a consistência interna da escala total e das subescalas; e (c) analisar a validade convergente da escala com outras medidas de interesse.

\section{MÉTODO}

\section{Participantes}

A amostra foi constituída por 222 participantes do sexo masculino da população geral com idades entre 18 e 65 anos $(M=28,61 ; D P=10,30)$, e com uma média $14,35(D P=2,55)$ anos de escolaridade. No que concerne ao estado civil, a maioria dos participantes reportou ser solteiro $(n=177 ; 79,7 \%), 39(17,6 \%)$ casado ou em união de facto, e 6 (2,7\%) eram divorciados. Em relação ao Índice de Massa Corporal (IMC) a amostra revelou uma média $(M=24,60 ; D P=$ $4,14)$ e uma distribuição equivalente à encontrada na população portuguesa para o sexo masculino, com 4 $(1,8 \%)$ participantes a apresentarem um IMC 
correspondente a magreza (IMC < 18,5), $137(61,71 \%)$ participantes um IMC normoponderal $(18,5 \leq \mathrm{IMC} \leq$ $25,0)$, e $81(36,49)$ participantes com excesso de peso ou obesidade $(\mathrm{IMC}>25,0)$.

\section{Instrumentos}

O protocolo de avaliação foi constituído por um breve questionário sociodemográfico (e.g., idade, sexo, anos de escolaridade, profissão, estado civil), pela versão portuguesa da MBAS-R e por três medidas de autorrespostas (validadas para a população masculina portuguesa) descritas seguidamente.

Índice de Massa Corporal (IMC). O IMC foi calculado através do índice de Quetelet, com base no peso e altura reportados pelos participantes.

Male Body Attitudes Scale- Revised (MBAS-R; Ryan et al., 2011). A MBAS-R é uma A MBAS-R é uma escala de autorresposta que resulta do processo de revisão da MBAS (Tylka et al., 2005) com o objetivo de avaliar atitudes de insatisfação e preocupação em relação à imagem corporal nos homens. A MBAS-R é composta por 15 itens divididos por 3 subescalas: massa muscular (MUS; 7 itens), massa gorda (GOR; 5 itens) e altura (ALT; 3 itens). Nesta medida é solicitado aos participantes que respondam a cada item usando uma escala de 5 pontos (de 1 = "Nunca" a 5 = "Sempre"), com valores mais elevados a corresponderem a graus superiores de insatisfação com a imagem corporal. Os valores de consistência interna ( $\alpha$ de Cronbach) da MBAS-R total e subescalas $\left(\alpha_{\text {TOTAL }}=0,88 ; \alpha_{\text {MUS }}=0,88 ; \alpha_{\mathrm{GOR}}=0,90 ; \alpha_{\mathrm{ALT}}=\right.$ 0,86), assim como os resultados da validade convergente, reportados no estudo original (Ryan et al., 2011), sugerem que a MBAS-R é uma medida fidedigna e um bom indicador das atitudes negativas dos homens em relação à sua aparência física. Esta escala constitui o principal alvo do presente estudo, pelo que a sua estrutura fatorial e qualidades psicométricas para a população portuguesa serão apresentadas na secção dos resultados.

Others as a Shamer - short version (OAS 2; Matos, Pinto-Gouveia, Gilbert, Duarte, \& Figueiredo, 2015). A OAS 2 é uma medida de autorrelato composta por 8 itens que avaliam a vergonha externa, i.e., a perceção de que os outros veem ou avaliam negativamente o sujeito (e.g., "As outras pessoas vêem-me como se eu fosse pequeno e insignificante"). Os participantes avaliam a frequência dessas experiências negativas, usando uma escala de 5 pontos $(0=$ "Nunca" a 4 = "Quase sempre"). A OAS 2 apresenta boas qualidades psicométricas na população portuguesa ( $\alpha$ de Cronbach $=0,82$ ). No presente estudo, a OAS 2 apresentou um alfa de Cronbach de 0,91.
Body Image Shame Scale (BISS; Duarte, PintoGouveia, Ferreira, \& Batista, 2015). A BISS é uma medida de autorrelato, composta por 14 itens, que visa avaliar a experiência de vergonha focada na imagem corporal. A escala inclui duas dimensões (com 7 itens cada): Vergonha corporal externa, que avalia a perceção de que os outros avaliam negativamente o indivíduo com base na sua imagem corporal (e.g., "Sinto-me desconfortável em situações sociais (por exemplo, jantares, festas) porque sinto que me podem criticar por causa da minha forma física."); Vergonha corporal interna, focada em autoavaliações negativas baseadas na imagem corporal (e.g., "Quando vejo o meu corpo ao espelho, sinto-me uma pessoa defeituosa"). Os participantes são solicitados a responder aos itens numa escala de 5 pontos (de $0=$ "Nunca" a $4=$ "Quase sempre"). No presente estudo foi utilizado o total da escala, calculado através do somatório das pontuações do total dos itens, em que resultados mais altos são indicadores de níveis mais elevados de vergonha em relação à imagem corporal. A BISS tem revelado boas qualidades psicométricas em amostras da população masculina (Duarte \& Ferreira, 2017). No presente estudo, a BISS revelou boa consistência interna com um alfa Cronbach de 0,95 .

Inflexible Eating Questionnaire (IEQ; Duarte, Ferreira, Pinto-Gouveia, Trindade, \& Martinho, 2017). O IEQ é uma medida composta por 11 itens que avaliam a inflexibilidade psicológica em relação ao padrão alimentar (e.g., "Fico preocupado quando não cumpro, mesmo que só ocasionalmente, as minhas regras alimentares."). Os participantes são solicitados a assinalar a sua concordância utilizando uma escala de resposta de 5 pontos (de 1 = "Discordo totalmente" a $4=$ "Concordo totalmente"), com resultados mais elevados a indicarem um padrão de maior inflexibilidade em relação às regras alimentares. O IEQ tem apresentado boas qualidades psicométricas, demonstrando boa consistência interna em amostras da população masculina ( $\alpha$ de Cronbach $=0,90$ ) (Duarte et al., 2017). No presente estudo esta medida apresentou um alfa de Cronbach de 0,91 .

\section{Procedimentos}

O procedimento relativo ao desenvolvimento da versão portuguesa da MBAS-R foi conduzido com base nas guidelines internacionais e de acordo com o método translate - translate back (Hill \& Hill, 2008). Assim, num primeiro momento, os itens da versão original em inglês foram traduzidos para português europeu por dois investigadores fluentes na língua inglesa e com conhecimentos sólidos na área da psicologia e da psicopatologia. Num segundo momento, esta versão 
portuguesa foi submetida a retroversão por um nativo da língua inglesa, com fluência na língua portuguesa. Finalmente, foi verificada a coerência da tradução dos itens e desenvolvida a versão final dos itens (ver Tabela 1).

Este estudo respeitou todas as normas éticas e os princípios deontológicos inerentes à investigação em psicologia, nomeadamente na recolha da amostra e tratamento dos dados. Após parecer favorável da Comissão de Ética e Deontologia da Faculdade de Psicologia e de Ciências da Educação da Universidade de Coimbra, a recolha de amostra foi realizada pelo método de amostragem exponencial não-discriminante de bola-de-neve. Foram endereçados convites a contactos dos investigadores, via email e através da rede social Facebook, aos quais foi solicitado a partilha por dois ou mais dos seus contactos. No convite à participação no estudo foi incluída informação acerca do estudo, o caráter voluntário e anónimo da participação e a confidencialidade dos dados obtidos e, ainda, os critérios de inclusão (sexo masculino, idades superiores a 18 anos, e nacionalidade portuguesa). Este convite online incluía um link que direcionava para a versão online do protocolo (na plataforma "LimeSurvey") após a obtenção do consentimento informado por escrito.

\section{Estratégia Analítica}

Análises Fatoriais Confirmatórias (AFC), com recurso ao software AMOS (v. 21; Analysis of a Moment Structures, SPSS Inc. Chicago, IL), foram conduzidas para testar a adequação da estrutura da MBAS-R com três e dois fatores/subescalas. O método de estimação do modelo utilizado foi o Método de Máxima Verosimilhança. O recurso a esta estratégia analítica deve-se ao facto de trabalhos anteriores terem indicado a plausibilidade tanto da solução com três fatores como com dois fatores (com a eliminação da subescala altura) (e.g., Ryan et al., 2011).

A qualidade global da AFC foi analisada através o teste de qui-quadrado $\left(\chi^{2}\right)$, no entanto e visto que teste estatístico é extremamente sensível ao tamanho da amostra (Schermelleh-Engel, Moosbrugger, \& Müller, 2003), foram igualmente selecionados outros indicadores recomendados relativamente independentes ao tamanho da amostra (Floyd \& Widaman, 1995): Qui-quadrado normativo (CMIN/g); Comparative Fit Index (CFI); TuckerLewis Index (TLI), Root Mean Square Error of Approximation (RMSEA). Considerou-se que valores de CFI e TLI entre 0,90 e 0,95 indicam um ajustamento adequado e valores superiores a 0,95 são indicadores de muito bom ajustamento dos dados (Brown, 2006; Kline, 2005). Em relação ao RMSEA, considerou-se um intervalo de confiança com $90 \%$, valores iguais ou inferiores a
0,08 são indicadores de bom ajustamento, e iguais ou inferiores a 0,10 de um ajustamento marginal (Arbuckle, 2008; Byrne, 2010; Hair, Black, Babin, \& Anderson, 2010).

$\mathrm{Na}$ análise da qualidade do ajustamento local do modelo foram examinados os pesos fatoriais $(\geq 0,40$; Tabachnick \& Fidell, 2007) e a fiabilidade individual dos itens $\left(R^{2} \geq 0,25\right.$; Marôco, 2010).

Para o estudo da qualidade dos itens foram utilizadas estatísticas descritivas (média e desvio-padrão) e análises de correlação entre cada item e a respetiva subescala, e com o total da MBAS-R. De acordo com as recomendações foram consideradas como aceitáveis correlações item-total (subescala e escala total) acima de 0,30 (Field, 2013). A consistência interna das subescalas e total da MBAS-R foi analisada através do alfa de Cronbach, considerando-se bons indicadores valores acima de 0,70 (Field, 2013). Foram, ainda, examinadas correlações de Pearson entre a MBAS-R (total e subescalas) e outras medidas de interesse (Cohen, Cohen, West, \& Aiken, 2003). Estas análises foram conduzidas com recurso ao software IBM SPSS Statistics (v. 21 SPSS; Armonk, NY: IBM Corp.).

\section{RESULTADOS}

\section{Estrutura Fatorial}

No Modelo 1 (três fatores: massa muscular, massa gorda e altura), os resultados da AFC demonstraram a inadequação do modelo, revelando um $\chi^{2}$ significativo $\left(\chi_{(87)}^{2}=373,507 ; p<0,001\right)$, e valores inaceitáveis nos restantes indicadores de ajustamento $(C M I N / g /=4,29$; $C F I=0,84 ; T L I=0,81 ;$ RMSEA $=0,12 ;$ I.C. $90 \%[0,11-$ $0,14]$ ). A análise dos índices de modificação indicou a pertinência de correlacionar os erros dos pares dos item8 e item-9, e item-5 e item- 6 , assim como do par do item-4 e item-7. A correlação entre estes erros é ainda suportada pela semelhança da construção gramática (entre o item-8 e item-9 e o item-5 e item-6), visto que os dois pares de itens começam de forma muito semelhante, e o par entre de item-4 e item-7 dado o conteúdo avaliado. Este procedimento resultou num melhoramento da qualidade do modelo $(\mathrm{CMIN} / g)=2,60$; $C F I=0,84 ; T L I=0,81 ;$ RMSEA $=0,12 ;$ I.C. $90 \%[0,11-$ $0,14]$ ), embora os dados revelem ainda um ajustamento não adequado deste modelo aos dados. Relativamente à qualidade de ajustamento local do modelo 1 , verificouse que todos os itens apresentam pesos fatoriais maiores ou iguais a 0,36 (item 15) e inferiores a 0,93 (item 13) e um $R^{2}$ entre 0,13 (item 15) e 0,87 (item 13). Os resultados confirmaram a fiabilidade de todos os itens, 
com exceção do item 15, o qual apresenta fraca fiabilidade individual $\left(R^{2}<0,25\right.$; Marôco, 2010).

No Modelo 2, a análise do ajustamento global da medida constituída por 12 itens e pelas respetivas subescalas massa muscular e massa gorda, indicou o ajustamento adequado aos dados $(\mathrm{CMIN} / \mathrm{g} /=2,51 ; \mathrm{CFI}=$ 0,95; TLI = 0,94; RMSEA = 0,08; I.C. 90\% [0,07 - 0,10]). $\mathrm{Na}$ análise do ajustamento local verificou-se que todos os itens apresentaram pesos fatoriais adequados, entre 0,47 (item 7) e 0,95 (item 11), isto é, superiores ao ponto de corte recomendado $(0,40$; Tabachnick \& Fidell, 2007). A fiabilidade dos itens foi igualmente confirmada pelos valores de $R^{2}$, os quais variaram entre 0,37 (item 4) e 0,90 (item 11) $\left(R^{2} \geq 0,25\right.$; Marôco, 2010) (Tabela 1).

\section{Tabela 1}

Estrutura final da versão portuguesa da MBAS-R, médias, desvio-padrão e correlação entre os itens e o total das respetivas subescalas e escala total $(\mathrm{N}=222)$

\begin{tabular}{|c|c|c|c|}
\hline Itens & $M(D P)$ & $\begin{array}{c}r \\
\text { item-total } \\
\text { subescala }\end{array}$ & $\begin{array}{c}r \\
\text { item-total } \\
\text { escala }\end{array}$ \\
\hline \multicolumn{4}{|l|}{ Subescala Massa Muscular } \\
\hline $\begin{array}{l}\text { 1. Eu acho que o meu corpo é } \\
\text { pouco musculado }\end{array}$ & $3,04(1,04)$ & $0,81^{* * *}$ & $0,60 * * *$ \\
\hline $\begin{array}{l}\text { 2. Eu acho que as minhas pernas } \\
\text { não são suficientemente } \\
\text { musculadas }\end{array}$ & $2,60(1,16)$ & $0,75^{* * *}$ & $0,51 * * *$ \\
\hline $\begin{array}{l}\text { 3. Eu acho que os meus braços } \\
\text { deveriam ser mais musculados }\end{array}$ & $3,20(1,05)$ & $0,77^{* * *}$ & $0,58^{* * *}$ \\
\hline $\begin{array}{l}\text { 4. Eu sinto-me envergonhado em } \\
\text { relação à minha massa } \\
\text { muscular }\end{array}$ & $1,99(1,05)$ & $0,71 * * *$ & $0,67^{* * *}$ \\
\hline $\begin{array}{l}\text { 5. Eu acho que as minhas costas } \\
\text { deveriam ser mais musculadas } \\
\text { (i.e., mais largas) }\end{array}$ & $2,58(1,07)$ & $0,77^{* * *}$ & $0,48^{* * *}$ \\
\hline $\begin{array}{l}\text { 6. Eu acho que o meu peito (i.e., } \\
\text { músculos peitorais) deveria ser } \\
\text { mais musculado }\end{array}$ & $2,86(1,16)$ & $0,79^{* * *}$ & $0,50^{* * *}$ \\
\hline $\begin{array}{l}\text { 7. Eu sinto-me satisfeito com a } \\
\text { massa muscular do meu corpo }\left(^{*}\right)\end{array}$ & $3,20(0,98)$ & $-0,60 * * *$ & $-0,54 * * *$ \\
\hline \multicolumn{4}{|l|}{ Subescala Massa Gorda } \\
\hline $\begin{array}{l}\text { 8. Eu acho que meu corpo deve } \\
\text { ser mais magro (i.e., mais seco) }\end{array}$ & $2,66(1,40)$ & $0,86^{* * *}$ & $0,62 * * *$ \\
\hline $\begin{array}{l}\text { 9. Eu acho que tenho demasiada } \\
\text { gordura no meu corpo }\end{array}$ & $2,50(1,29)$ & $0,90^{* * *}$ & $0,71^{* * *}$ \\
\hline $\begin{array}{l}\text { 10. Comer doces, bolos, ou outros } \\
\text { alimentos muito calóricos faz- } \\
\text { me sentir gordo }\end{array}$ & $2,17(1,26)$ & $0,81 * * *$ & $0,63 * * *$ \\
\hline $\begin{array}{l}\text { 11. Eu sinto-me excessivamente } \\
\text { gordo }\end{array}$ & $1,83(1,19)$ & $0,91 * * *$ & $0,74 * * *$ \\
\hline $\begin{array}{l}\text { 12. Ver o meu reflexo (no espelho } \\
\text { ou num vidro) faz-me sentir mal } \\
\text { com a minha gordura corporal }\end{array}$ & $1,91(1,16)$ & $0,87^{* * *}$ & $0,76^{* * *}$ \\
\hline
\end{tabular}

Os resultados obtidos nas análises fatoriais confirmatórias revelaram a adequação da estrutura com 2 fatores/subescalas, com a eliminação da subescala altura (i.e., dos itens 13, 14 e 15) para a versão portuguesa da MBAS-R. A decisão da eliminação destes itens e respetiva subescala foi ainda corroborada pela análise dos valores da correlação entre estes itens e o total da escala (item 13: "Eu desejava ser mais alto" =0,40; item 14: "Eu sinto-me satisfeito com a minha altura" = -0,24; item 15: "Eu sinto vergonha da minha altura" $=0,35$ ). Análises posteriores relativas às propriedades psicométricas da versão portuguesa da MBAS-R serão avaliadas para a versão com 12 itens (dois fatores/subescalas).

\section{Análise dos itens e consistência interna}

O estudo das características dos 12 itens que constituem a versão portuguesa da MBAS-R foi realizado através de análises descritivas (média e desvio-padrão) e de correlação entre cada item e a respetiva subescala, e com a escala total (Field, 2013; Tabachnick \& Fidell, 2007). Os resultados permitiram verificar que todos os itens apresentam correlações item-subescala iguais ou superiores a -0,60 (item 7) e item-total da escala iguais ou superiores a 0,50 (item 6), indicando que os itens contribuem consistentemente para a respetiva subescala e para a medida global (Tabela 1). O coeficiente de alfa de Cronbach obtido para a escala total $(\alpha=0,87)$ é indicador de uma boa consistência interna (Field, 2013; Tabachnick \& Fidell, 2007). Em relação às duas dimensões teóricas que a compõem, verificou-se igualmente uma adequada consistência interna: 0,87 para a subescala Massa Muscular e 0,92 para a subescala Massa Gorda.

\section{Validade convergente}

O estudo da validade convergente da versão portuguesa da MBAS-R foi realizado através de correlações de Pearson entre o seu total e subescalas e medidas de vergonha externa (OAS 2), vergonha corporal (BISS), e inflexibilidade em relação ao padrão alimentar (IEQ).

\section{Tabela 2}

Correlação entre MBAS-R total e subescalas e outras medidas de interesse $(N=222)$

\begin{tabular}{lllll}
\hline MBAS-R & OAS 2 & BISS & IEQ & IMC \\
\hline Total & $0,32^{* * *}$ & $0,64^{* * *}$ & $0,38^{* * *}$ & $0,29 * * *$ \\
\hline SMM & $0,26^{* * *}$ & $0,38^{* * *}$ & $0,18^{* *}$ & $-0,14^{*}$ \\
\hline SMG & $0,22^{* * *}$ & $0,62^{* * *}$ & $0,43^{* * *}$ & $0,58^{* * *}$ \\
\hline
\end{tabular}

Nota. MBAS-R Total e subescalas = Male Body Attitudes Scales-Revised; SMM = Subescala Massa Muscular; SMG = Subescala Massa Gorda; OAS $2=$ Others as a Shamer-short version; BISS = Body Image Shame Scale; IEQ = Inflexible Eating Questionnaire; IMC = Índice de Massa Corporal.

${ }^{*} p<0,05$; ** $p<0,01$; ** $p<0,001$. 
Os resultados (Tabela 2) demonstraram associações positivas, com magnitudes de baixas a moderadas, entre MBAS-R total e subescalas e a medida de vergonha externa (OAS 2). Em relação à medida de vergonha corporal (BISS) verificou-se uma associação positiva forte tanto com a MBAS-R total, como com a subescala massa gorda, e moderada com a subescala massa muscular. No que concerne à inflexibilidade alimentar (IEQ), os resultados revelaram associações positivas com magnitudes moderadas com a escala total e subescala massa gorda, e com magnitude fraca com a subescala massa muscular. Finalmente, o IMC apresentou associações positivas com a MBAS-R total e a subescala massa gorda (com magnitudes moderada e forte, respetivamente) e uma associação negativa e fraca com a subescala massa muscular.

\section{DISCUSSÃO E CONCLUSÃO}

A literatura documenta que a imagem corporal é uma dimensão importante para a auto e heteroavaliação na população do sexo masculino (McCabe \& Ricciardelli, 2001) com impacto significativo em diferentes indicadores de psicopatologia (Calogero, 2009; Dakanalis \& Riva, 2013; Dakanalis et al., 2015; Sepúlveda et al., 2016). Diversos autores salientam, no entanto, a inadequação do uso de medidas desenvolvidas para as mulheres para a compreensão da vivência da imagem corporal em homens (e.g., Cafri \& Thompson, 2004; McCabe \& Ricciardelli, 2001; Ridgeway \& Tylka, 2005; Ryan et al., 2011). Neste contexto, Tylka et al. (2005) desenvolveram a Male Body Attitudes Scales (MBAS) que visa medir as preocupações e as atitudes masculinas em relação à aparência física. Posteriormente, Ryan et al. (2011), tendo em vista a superação das limitações apontadas a esta escala, desenvolveram uma versão revista (MBAS-R - Male Body Atitudes Scales-Revised). Embora a MBAS-R tenha revelado ser uma medida robusta, fidedigna e útil (Ryan et al., 2011), os autores desta medida recomendam a análise da validade e utilidade deste instrumento em línguas diferentes da língua inglesa, assim como da adequação da sua estrutura fatorial através de metodologias estatísticas robustas. Assim, o presente estudo teve como objetivos: (1) desenvolver a versão portuguesa da MBAS-R; (2) testar a adequação da estrutura com três e duas subescalas; e (3) examinar as suas qualidades psicométricas numa amostra de homens da população geral portuguesa.

Em relação ao primeiro objetivo, no processo de tradução e adaptação da MBAS-R foram seguidas as diretrizes recomendadas de modo a minimizar os erros de tradução/adaptação e a garantir que deste processo resultasse uma versão portuguesa equivalente à medida original (Hill \& Hill, 2008).

No que concerne à estrutura fatorial da MBAS-R, e atendendo aos dados da literatura acerca da MBAS (Blashill \& Vander Wal, 2009; Sepúlveda et al., 2016; Tylka et al., 2005), foram testados dois modelos distintos: Modelo 1, com três fatores (massa muscular, massa gorda e altura); e Modelo 2, com dois fatores (massa muscular e massa gorda) através de uma série de análises fatoriais confirmatórias. Os resultados das AFC do presente estudo dão suporte unicamente à estrutura de dois fatores, composta pelas subescalas referentes à avaliação da insatisfação acerca da massa muscular e da massa gorda. Estes resultados da versão portuguesa da MBAS-R são consistentes com os dados obtidos na validação da versão da MBAS para a língua espanhola para a população adolescente, os quais suportam a eliminação da subescala altura (Sepúlveda et al., 2016). Adicionalmente, os resultados do presente estudo são congruentes com Ryan et al. (2011) que apontaram as limitações do uso desta subescala altura e com Blashill \& Vander Wal (2009) que mostraram que o modelo da MBAS composto pelas duas subescalas (massa muscular e massa gorda) apresenta uma adequação mais forte, comparativamente à versão original de três subescalas. Em síntese, os resultados indicaram a adequação da versão portuguesa da MBAS-R, constituída por 12 itens divididos em duas dimensões (massa muscular e massa gorda).

Os valores de alfas de Cronbach da versão portuguesa da MBAS-R obtidos tanto para a escala total como para as subescalas massa muscular e massa gorda são indicadores de boa consistência interna e similares aos reportados por Ryan et al. (2011).

De modo a confirmar a validade e a utilidade da escala para avaliar a insatisfação com a imagem corporal em homens, foram exploradas as associações entre a MBAS-R total e subescalas e medidas de vergonha externa, vergonha corporal e de inflexibilidade psicológica em relação à alimentação. Os resultados mostraram que a insatisfação corporal nos homens, avaliada pela MBAS-R, se associa positivamente a sentimentos de vergonha e sentimentos de inferioridade. Estes resultados parecem suportar dados de estudos prévios que documentam uma associação, tanto em amostras do sexo masculino como feminino, entre insatisfação com a imagem corporal e um sentimento geral de desvalorização e de inferioridade (Duarte \& Pinto-Gouveia, 2016). Tal como esperado, esta 
associação é mais forte quando estes sentimentos de inadequação e inferioridade são percecionados como baseados na imagem corporal dos participantes, tal como avaliado pela Body Image Shame Scale. Adicionalmente, os resultados revelaram que graus mais elevados de insatisfação corporal (MBAS-R) se associam a maior inflexibilidade em relação às regras alimentares. Embora este seja o primeiro estudo que explora a relação entre insatisfação com a imagem corporal e a adoção de regras rígidas em relação ao plano alimentar em homens, os dados deste estudo parecem corroborar que uma apreciação negativa da aparência física tende a associarse a atitudes e comportamentos alimentares maladaptativos (Calogero, 2009; Dakanalis \& Riva, 2013; Dakanalis et al., 2015; Sepúlveda et al., 2016; Smith et al., 2011),

No sentido de clarificar a relação entre peso corporal e insatisfação com a imagem corporal na população do sexo masculino foram exploradas as relações entre IMC e o total e subescalas da MBAS-R. Os dados obtidos revelam que os homens que com IMC superiores tendem a reportar níveis globais de insatisfação corporal e particularmente graus mais altos de insatisfação em relação à sua massa gorda. No entanto, foi observada uma relação negativa entre IMC e apreciação da massa muscular, isto é, sujeitos com IMCs mais baixos tendem a reportar níveis superiores de insatisfação em relação à massa muscular e desejo de ganhar massa corporal. Estes resultados parecem corroborar que na população do sexo masculino não existe uma relação linear positiva entre IMC e insatisfação com a imagem corporal, em contraste com o que é encontrado em amostras do sexo feminino (Dakanalis \& Riva, 2013; Yean et al., 2013).

Este estudo parece ser um importante contributo para o desenvolvimento e validação de medidas para o estudo da imagem corporal na população masculina. No entanto os resultados apresentados devem ser interpretados tendo em conta algumas limitações. De facto, este é o primeiro estudo que examina a validade da versão portuguesa da MBAS-R, sendo recomendável que estudos futuros comprovem a adequação da sua estrutura fatorial e da qualidade dos itens em diferentes amostras da população masculina de língua portuguesa. Futuras investigações poderão, ainda, analisar o comportamento desta escala em amostras masculinas específicas (por exemplo, em adolescentes). Outra limitação importante, diz respeito ao facto deste estudo não analisar a validade temporal desta medida. Trabalhos futuros deverão assim examinar a estabilidade temporal das dimensões avaliadas pela MBAS-R. Adicionalmente, e não obstante a fraca adequação da subescala altura ter sido apontada por estudos prévios
(Blashill \& Vander Wal, 2009; Tylka et al., 2005), futuros estudos deverão clarificar se as limitações associadas a esta subescala (mais especificamente, a fraca adequação da subescala altura e o fraco comportamento dos itens dessa dimensão, traduzido pelos valores baixos de correlação com o total da escala) se devem ao número e/ou qualidade dos itens utilizados para avaliar esta dimensão ou se os resultados obtidos são intrínsecos ao constructo de altura e à fraca relação entre esta dimensão e a vivência de insatisfação corporal na população masculina.

Em conclusão, este estudo demonstra que a versão portuguesa da MBAS-R, constituída por 12 itens, é uma medida breve, válida e com boas qualidades psicométricas para avaliar a insatisfação com a imagem corporal no sexo masculino e contribui para colmatar a lacuna existentes no estudo desta dimensão, dos seus correlatos e impacto psicossocial na população do sexo masculino.

Conflito de interesses | Conflict of interest: Nenhum | None. Fontes de financiamento | Funding sources: Nenhuma | none.

\section{REFERÊNCIAS}

Arbuckle, J. L. (2008). AMOS 17.0 User's Guide. Chicago, IL: SPSS Retrieved from http://dspace.utalca.cl/bitstream/1950/9420/1/Amos17.0-User\%27s-Guide.pdf

Blashill, A. J. (2010). Elements of male body image: Prediction of depression, eating pathology and social sensitivity among gay men. Body Image, 314), 310-316. https://doi.org/10.1016/j.bodyim.2010.07.006

Blashill, A. J., \& Vander Wal, J. S. (2009). The Male Body Attitudes Scale: A confirmatory factor analysis with a sample of gay men. Body Image, 6(4), 322-325. https://doi.org/10.1016/j.bodyim.2009.07.004

Brown, T. A. (2006). Confirmatory factor analysis for applied research. New York, NY: Guildford Press.

Byrne, B. M. (2010). Structural equation modelling with AMOS: Basic concepts, applications, and programming (2nd ed.). New York, NY: Routledge.

Cafri, G., Strauss, J., \& Thompson, J. K. (2002). Male body image: Satisfaction and its relationship to well-being using the somatomorphic matrix. International Journal of Men's Health, 1(2), 215-231. https://doi.org/10.3149/jmh.0102.215

Cafri, G., \& Thompson, J. K. (2004). Measuring male body image: A review of the current methodology. Psychology of Men and Masculinity, 5(1), 18-29. https://doi.org/10.1037/1524-9220.5.1.18

Cain, A. S., Epler, A. J., Steinley, D., \& Sher, K. J. (2011). Concerns related to eating, weight, and shape: Typologies and transitions in men during the college years. International Journal of Eating Disorders, 45(6), 1-8. https://doi.org/10.1002/eat.20945

Calogero, R. M. (2009). Objectification processes and disordered eating in British women and men. Journal of Health Psychology, 14(3), 394-402. https://doi.org/10.1177/1359105309102192

Cohane, G. H., \& Pope Jr., H. G. (2001). Body image in boys: A review of the literature. International Journal of Eating Disorders, 29(4), 373-379. https://10.1002/eat.1033

Cohen, J., Cohen, P., West, S. G., \& Aiken, L. S. (2003). Applied multiple regression/correlation analysis for the behavioral sciences (3rd ed.). Mahwah, NJ: Erlbaum. 
Cooper, P. J., Taylor, M. J., Cooper, Z., \& Fairbum, C. G. (1987). The development and validation of the Body Shape Questionnaire. International Journal of Eating Disorders, 6(4), 485-494. https://doi.org/10.1002/1098-108X(198707)6:4<485::AIDEAT2260060405>3.0.CO;2-O

Daniel, S., \& Bridges, S. K. (2010). The drive for muscularity in men: Media influences and objectification theory. Body Image, 7(1), 32-38. https://doi.org/10.1016/j.bodyim.2009.08.003

Dakanalis, A., \& Riva, G. (2013). Current considerations for eating and body-related disorders among men. In L. B. Sams \& J. A. Keels (Eds.), Handbook on body image: Gender differences, sociocultural influences and health implications (pp. 195-215). New York, NY: Nova Science Publishers.

Dakanalis, A., Zanetti, A. M., Riva, G., Colmegna, F., Volpato, C., Madeddu, F., \& Clerici, M. (2015). Male body dissatisfaction and eating disorder symptomatology: Moderating variables among men. Journal of Health Psychology, 20(1), 80-90. https://doi.org/10.1177/1359105313499198

Duarte, C., \& Ferreira, C. (2017). The need to assess body image shame in men: Confirmatory factor analysis and psychometric properties of the Body Image Shame Scale in a male population. Manuscript submitted for publication.

Duarte, C., Ferreira, C., Pinto-Gouveia, J., Trindade, I. A., \& Martinho, A. (2017). What makes dietary restraint problematic? Development and validation of the Inflexible Eating Questionnaire. Appetite, 114, 146-154. https://doi.org/10.1016/j.appet.2017.03.034

Duarte, C., \& Pinto-Gouveia, J. (2016). Self-defining memories of body image shame and binge eating in men and women: Body image shame and self-criticism in adulthood as mediating mechanisms. Sex Roles, 77(5-6), 338-351. https://doi.org/10.1007/s11199-016$0728-5$

Duarte, C., Pinto-Gouveia, J., Ferreira, C., \& Batista, D. (2015). Body image as a source of shame: A new measure for the assessment of the multifaceted nature of body image. Clinical Psychology and Psychotherapy, 22(6), 656-666. https://doi.org/10.1002/cpp.1925

Fairburn, C. G., \& Beglin, S. J. (1994). Assessment of Eating Disorders: Interview or self-report questionnaire? International Journal of Eating Disorders, 16(4), 363-370.

Field, A. (2013). Discovering statistics using IBM SPSS Statistics (4th ed.). London: Sage. Retrieved from http://www.fb4all.com/download/ebooks/statistics/\%23Discoveri ng\%20Statistics\%20Using\%20SPSS\%202013.pdf

Floyd, F. J., \& Widaman, K. F. (1995). Factor analysis in the development and refinement of clinical assessment instruments. Psychological Assessment, 7(3), 286-299. https://doi.org/10.1037/1040-3590.7.3.286

Garner, D. M., Olmsted, M. P., \& Polivy, J. (1983). Development and validation of multidimensional Eating Disorder Inventory for anorexia nervosa and bulimia. International Journal of Eating Disorders, 2(2), 15-34. https://doi.org/10.1002/1098108X(198321)2:2<15::AID-EAT2260020203>3.0.CO;2-6

Hair, J. F., Black, W. C., Babin, B. J., \& Anderson, R. E. (2010). Multivariate data analysis (7th ed.). New York, NY: Pearson.

Hill, M. M., \& Hill, A. (2008). Investigação por questionário [Investigation by questionnaire] ( $2 \mathrm{nd}$ ed.). Lisboa: Edições Sílabo.

Kline, R. B. (2005). Principles and practice of structural equation modeling (2nd ed.). New York, NY: Guilford Press.

Marôco, J. (2010). Análise Estatística com o PASW Statistics [Statistical Analysis with PASW statistics]. Lisboa: Report Number.
Matos, M., Pinto-Gouveia, J., Gilbert, P., Duarte, C., \& Figueiredo, C. (2015). The Other as Shamer Scale - 2: Development and validation of a short version of a measure of external shame. Personality and Individual Differences, 74, 6-11. https://doi.org/10.1016/j.paid.2014.09.037

McCabe, M. P., \& Ricciardelli, L. A. (2001). Body image and body change techniques among young adolescent boys. European Eating Disorders Review, 9(5), 335-347. https://doi.org/10.1002/erv.389

McCreary, D. R., \& Sasse, D. K. (2000). An exploration of the drive for muscularity in adolescent boys and girls. Journal of American College Health, 48(6), 297-304. https://doi.org/10.1080/07448480009596271

Olivardia, R., Pope, H. G., Borowiecki, J. J., \& Cohane, G. H. (2004). Biceps and body image: The relationship between muscularity and self-esteem, depression, and eating disorder symptoms. Psychology of Men and Masculinity, 5(2), 112-120. https://doi.org/10.1037/1524-9220.5.2.112

Pope, H., Phillips, K. A., \& Olivardia, R. (2000). The Adonis Complex: The secret crisis of male body obsession. New York, NY: Free Press.

Ridgeway, R., \& Tylka, T. L. (2005). College men's perceptions of ideal body composition and shape. Psychology of Men and Masculinity, 6(3), 209-220. https://doi.org/10.1037/15249220.6.3.209

Ryan, T. A., Morrison, T. G., Roddy, S., \& McCutcheon, J. (2011). Psychometric properties of the Revised Male Body Attitudes Scale among Irish men. Body Image, 8(1), 64-69. https://doi.org/10.1016/j.bodyim.2010.10.004

Schermelleh-Engel, K., Moosbrugger, H., \& Müller, H. (2003). Evaluating the fit of structural equation models: Tests of significance and descriptive goodness-of-fit measures. Methods of Psychological Research Online, 8(2), 28-74. Retrieved from https://www.researchgate.net/publication/251060246_Evaluating _the_Fit_of_Structural_Equation_Models_Tests_of_Significance_a nd_Descriptive_Goodness-of-Fit_Measures

Sepúlveda, A. R., Anastasiadou, D., Pellegrin, Y., Parks, M. Blanco, M., Garcia, P., \& Bustos, C. (2016). Confirmatory factor analysis of the Male Body Attitudes Scale (MBAS) among Spanish adolescent males. Men and Masculinities, 20(3), 345-363. https://doi.org/10.1177/1097184X16634798

Smith, A. R., Hawkeswood, S. E., Bodell, L. P., \& Joiner, T. E. (2011). Muscularity versus leanness: An examination of body ideals and predictors of disordered eating in heterosexual and gay college students. Body Image, 8(3), 232-236. https://doi.org/10.1016/j.bodyim.2011.03.005

Stanford, J. N., \& McCabe, M. P. (2002). Body image ideal among males and females: Sociocultural influences and focus on different body parts. Journal of Health Psychology, 7(6), 675-684. https://doi.org/10.1177/1359105302007006871

Tabachnick, B. G., \& Fidell, L. S. (2007). Using multivariate statistics (5th ed.). Boston: Pearson.

Tylka, T. L., Bergeron, D., \& Schwartz, J. P. (2005). Development and psychometric evaluation of the Male Body Attitudes Scale (MBAS). Body Image, 2(2), 161-175. https://10.1016/j.bodyim.2005.03.001

Yean, C., Benau, E. M., Dakanalis, A., Hormes, J. M., Perone, J., \& Timko, C. A. (2013). The relationship of sex and sexual orientation to self-esteem, body shape satisfaction, and eating disorder symptomatology. Frontiers in Psychology, 4. https://doi.org/10.3389/fpsyg.2013.00887 